\title{
Multi-scale Modeling of Trauma Injury
}

\author{
Celina Imielinska ${ }^{1}$, Andrzej Przekwas ${ }^{2}$, and X.G. Tan ${ }^{2}$ \\ ${ }^{1}$ Dept. of Biomedical Informatics and Dept. of Computer Science, \\ Columbia University, \\ 701 W. $168^{\text {th }}$ Str. HHSC 201, New York, NY 10032 \\ ci42@columbia.edu \\ ${ }^{2}$ CFD Research Corp., 215 Wynn Drive, Huntsville, AL 35505 \\ $\{a j p, x g t\} @_{c} f d r c . c o m$
}

\begin{abstract}
We develop a multi-scale high fidelity biomechanical and physiologically-based modeling tools for trauma (ballistic/impact and blast) injury to brain, lung and spinal cord for resuscitation, treatment planning and design of personnel protection. Several approaches have been used to study blast and ballistic/impact injuries. Dummy containing pressure sensors and synthetic phantoms of human organs have been used to study bomb blast and car crashes. Large animals like pigs also have been equipped with pressure sensors exposed to blast waves. But these methods do not anatomically and physiologically biofidelic to humans, do not provide full optimization of body protection design and require animal sacrifice. Anatomy and medical image based high-fidelity computational modeling can be used to analyze injury mechanisms and to optimize the design of body protection. This paper presents novel approach of coupled computational fluid dynamics (CFD) and computational structures dynamics (CSD) to simulate fluid (air, cerebrospinal fluid) solid (cranium, brain tissue) interaction during ballistic/blast impact. We propose a trauma injury simulation pipeline concept staring from anatomy and medical image based high fidelity 3D geometric modeling, extraction of tissue morphology, generation of computational grids, multiscale biomechanical and physiological simulations, and data visualization.
\end{abstract}

\section{Introduction}

Primary blast injury (PBI) results from an interaction of pressure wave with the human body, and gas filled organs, ear, lungs, and gastrointestinal track. A typical explosion creates a shock wave traveling at three to five times the speed of sound. Temperature across the shock wave can increase over 1000 degrees Celsius near the explosion and pressure can increase abruptly to more than 20 atmospheres, Fig. 1 . Closed spaces such as rooms and street canyons cause reflections and shock wave interference patterns that can amplify pressure changes. A large percentage of fatal blast injuries result from PBIs, in which the shock wave directly damages the lungs through violent, localized pressure changes. Because a shock wave travels faster in liquids and solids than air, organs like the lungs, intestines, and inner ear are damaged by shear stresses when a shock wave reaches tissue-gas interfaces. The lungs are most 
vulnerable to PBIs because they contain large surface areas of fragile alveoli where oxygen is exchanged for carbon dioxide. Differences in wave propagation mechanics at the interface between air and blood in alveolar membranes cause large deformations of lung tissue that collapse alveolar sacs, tear alveolar membranes and rupture blood vessels. The extent of lung injury is a decisive parameter for mortality in victims surviving an explosion. Because the symptoms of primary blast injury are often delayed, victims may not receive timely treatment.

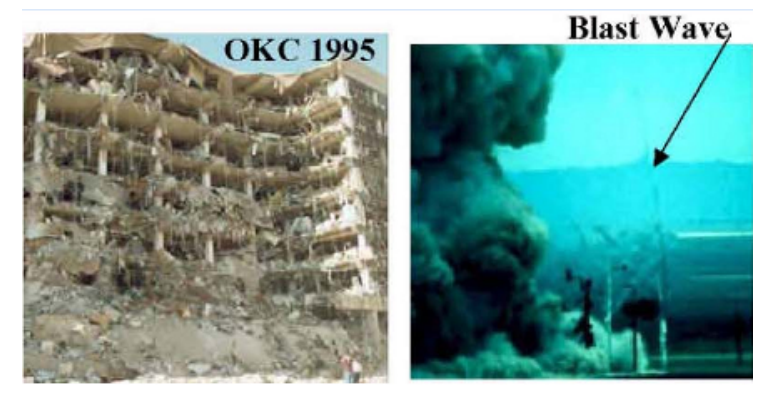

Fig. 1. Blast wave dynamics - injury potential

We are seeking to mathematically model blast wave interaction with human body and ballistic/impact injury to the vital human organs. This modeling effort requires a combination of diverse disciplines including gas and human body dynamics, tissue biomechanics, and the pathophysiology of organ damage.

\section{Material and Methods}

We demonstrate a 3D modeling pipeline starting with acquisition of data from various imaging modalities that can provide the most detailed anatomy, to high fidelity FEM modeling. This high-fidelity simulation combining right mathematical models describing body dynamics, tissue biomechanics, and injury pathophysiology requires detailed geometry and material properties of the organs (torso/lung, head/brain). Such information has to be extracted from anatomical data and multimodal medical imaging. Computational models have to be calibrated and validated against available experimental models such as physical surrogates and animal "models". The computational model will be invaluable in better understanding of injury mechanisms, in better planning of experimental study, and in optimized design of personnel protection armor.

\subsection{Design of Imaging System for Organ Injury}

We define a 3D imaging pipeline that provides image acquisition, processing, segmentation, and modeling of 3D anatomy of human anatomical structures. The images come from CT, MR patient scans and also from CT and color cryosections of the Visible Human datasets. Methods for function and physiology are applied to depict, assess and classify the full extent of brain, spine, and lung injury under trauma. The structure are later used for FEM impact and biomechanics simulations. 


\subsection{Modeling Head Anatomy}

We use two sets of data depicting head anatomy:

(a) the Visible Human Male color cryosection data segmented, Fig. 2, and reconstructed/rendered with 3D Vesalius Visualizer [1], Fig. 3.

(b) Visible Human Male CT, Fig. 4, segmented with hybrid segmentation method [2,3], Fig. 5., and reconstructed with t-shells [4], Fig. 6.

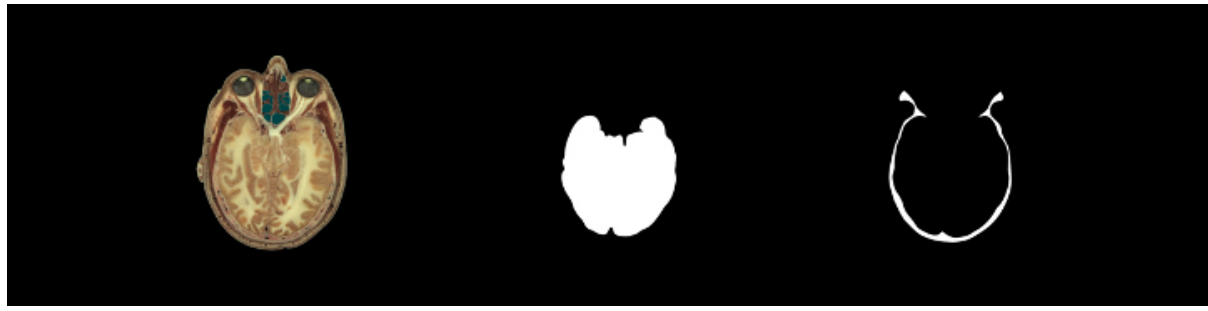

(a)

(b)

(c)

Fig. 2. (a) Visible Human data with (a) brain and (b) skull regions segmented

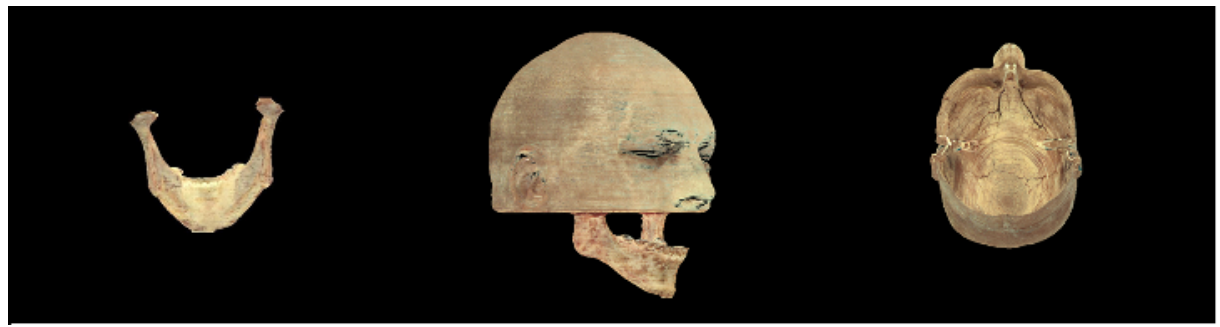

Fig. 3. Selected 3D models from the Visible Human Make data visualizes with 3D Vesalius Visualizer [1]
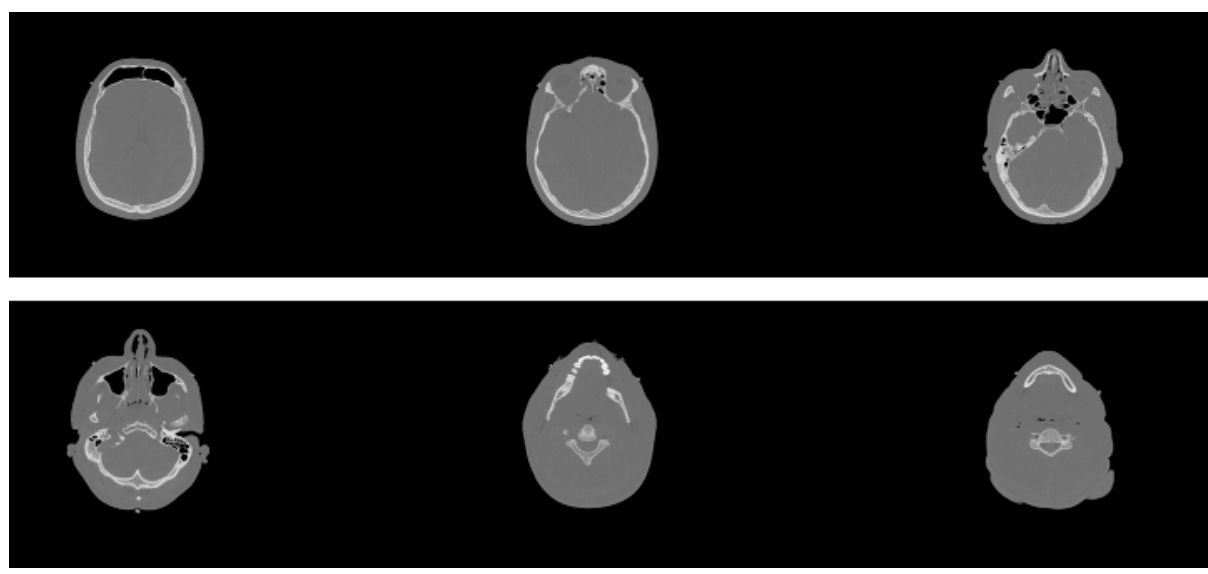

Fig. 4. Visible Human Male CT input data 

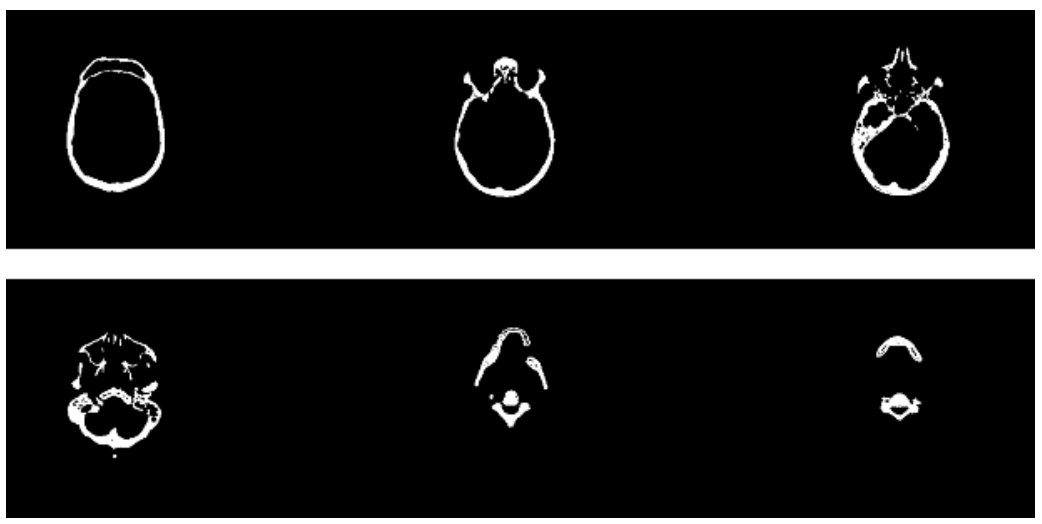

Fig. 5. Visible Human Male CT segmented data

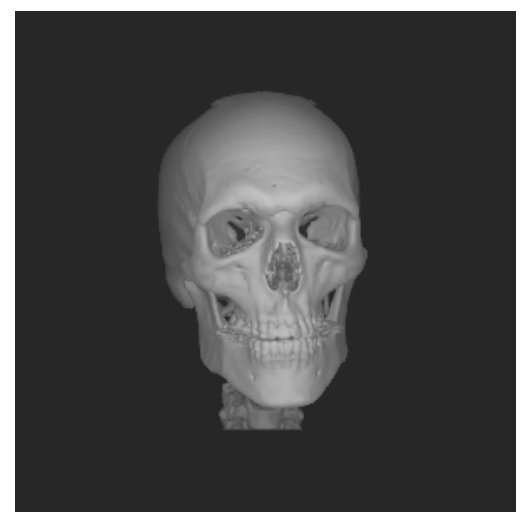

Fig. 6. Visible Human Male skull reconstructed with t-shells. [3].

\subsection{Geometry Preserving Automated Coarsening of Triangulated Surfaces}

High resolution medical images and image processing software generate large number of surface triangles. This is desired for the image visualization but is not practical for FEM biomechanics modeling. It is not uncommon to see millions of triangles on the cranium and brain surfaces. A FEM tetrahedral volume mesh generated from such number of surface mesh would have hundreds of millions elements. We have developed a novel algorithm and a software module for automated coarsening of triangulated surfaces while preserving the geometrical fidelity. We have tested it on automated coarsening of the surface triangle meshes for a human head depicted in Fig. 7. Figures 6a-c present the original human head mesh with 950,000 triangles and two levels of coarsened grids. The algorithm provides very powerful capability of generating coarse tessellation which can be used directly for tetrahedral meshing or as a surface geometry for generation of NURB surfaces and further high quality structured FEM grids. In the future, we plan to implement this module into a data processing software framework for semi-automated generation of high quality FEM grids for human body, with emphasis on human head, neck, and spine. 


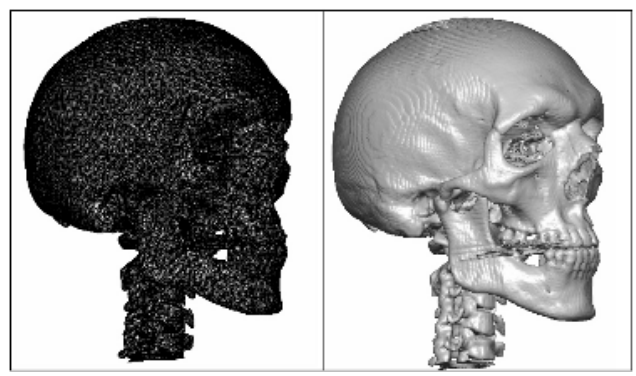

(a)

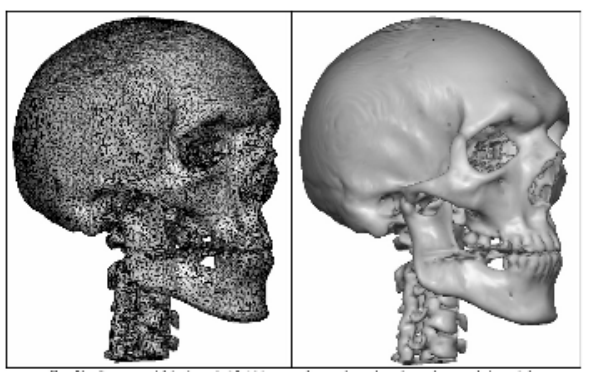

(b)

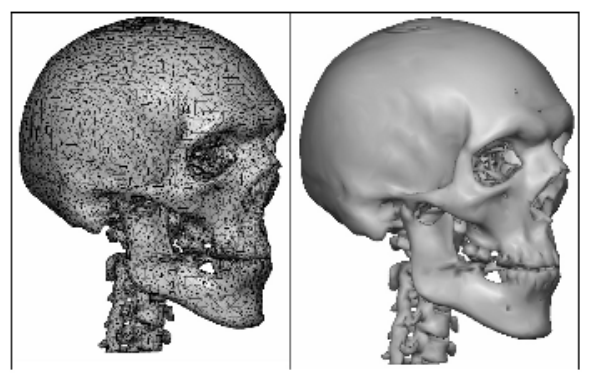

(c)

Fig. 7. (a) original model with 950,000 triangles (too fine to see) and rendered solid model, (b) coarsened mesh with 95,000 triangles and rendered resultant solid model, (c) coarsened mesh with 37,500 triangles and rendered resultant model

\subsection{Adaptation of FEM Model for Ballistic Injury}

CFDRC is developing software tools FEM-Bi, for multiscale modeling of blast injuries to thorax, lungs, brain, and spine coupled to physiological trauma injury model CoBi. We are adapting FEM-Bi for modeling ballistic impact of head protective gear materials in proximity to animal/human head and for modeling head injury. The FEM model can be validated on existing experimental data of mechanical behavior of material used in the protective gear and protection padding.

\subsection{Explicit Dynamics Systems}

In the current helmeted head model, larger number of finite elements (100K or more) is needed to properly resolve the complex geometry and stress/strain localization. As a result, the solution time for the implicit FEM solution for large grid counts ( $>100 \mathrm{~K}$ ) will increase dramatically and become prohibitively expensive. To speed up the computations and take less computer memory, we developed an explicit solver for the FEM equations. For large-scale high-speed event, it is advantageous to use the conditional-stable explicit solver because:

1) A small time step is required to track the high-frequency portion of the response anyway, making it impossible to take advantage of the large time steps permitted by implicit methods; 
2) Time integration of the discrete momentum equations does not require the solution of any equations due to the use of diagonal mass matrix. The construction and solving of linear system in the implicit method in each iteration of time step is very time-consuming, especially for the large finite element model;

3) Robustness for the nonlinear problem compared to the Newton iterations in nonlinear implicit solver, in which the convergence failure can often occur due to 'rough' events such as contact-impact or the large time step.

\subsection{Explicit Solution of Frictional Contact Problem}

The development includes the virtual work and contact searching, which appears to be highly desirable for the general robustness of explicit finite element techniques. Since the explicit formulation has no convergence problem caused by the nonlinear iterations, the resulting frictional contact model is suitable for applications displaying significant non-linear behavior. An important aspect of contact problems is the method used to convert the associated variational inequality to an equality suitable for finite element solution. In the explicit analysis we still adopt the penalty method since it offers distinct advantages on the integration algorithm for the frictional tractions, compared to the Lagrange multipliers method. At beginning of each time step, the estimate for an appropriate choice of the penalty parameter is based on the material properties and geometric size of the shell element. The increasing of the default penalty parameter becomes necessary if the results show visible penetration, and thus does not fulfill the constraint equation in a correct way. On the other hand, the larger penalty parameter may decrease the explicit time step size.

The most important practical aspect of computing the contact force vector is acquisition of the projection for the finite element node, i.e., the contact searching. Briefly stated, the algorithm consists of the following three phases: 1) Identification of the closest target node for the FE node; 2) Among all target elements sharing the closest node, identify the element containing the projection; 3) Calculate the coordinates of the projected point. In the current implementation, we calculate the closest target node once at the beginning of each global time step, which is used for the fluid domain in the fluid-structure interaction (FSI) simulation. During one global time step the local search for the projection is conducted for each FE explicit time step.

\subsection{Parametric Simulations}

We will use in the future the medical imaging data and resulting 3D models to set-up parametric model of the head, spine and other anatomical regions. We will perform ballistic impact FEM simulations and analyze the dynamics of protective gear in contact with underlying anatomy, the material-structure impact loads and potential injury to the anatomical structure(s).

\section{Results}

In the following we show examples to verify the correctness of the new developed explicit finite element solver, and the newly developed truss model used in the gear protected head under impact. 


\subsection{Test on Explicit Finite Element Solver}

We have implemented the explicit solver of the finite element model for the FSI applications, in addition to the existing implicit solver. Here are some preliminary results to show the effectiveness of the developed explicit solver.

The key features of the current explicit solver implementation include:

1) Optimal explicit time step size to maintain numerical stability and accuracy,

2) Global/local search algorithm for the contact problem,

3) Full-integrated element to avoid the hourglassing modes,

4) Numerical dissipation for the central difference time stepping.

The new explicit formulation for the structures calculations was then tried out on a large model with 44,791 eight-node solid element (155,394 dofs) as shown in Fig. 8. We made the FE mesh from the geometry data provided by [5]. The FE takes into account the cerebrum, cerebellum, falx and tentorium, CSF, dura, 3 layered cortical and trabecular skull bone, scalp, and facial bone. Table 1 shows the CPU time and computer memory needed for the test case, in which the head is moving forward with an initial velocity. The stable time step size for the explicit algorithm is calculated internally and adjusted.
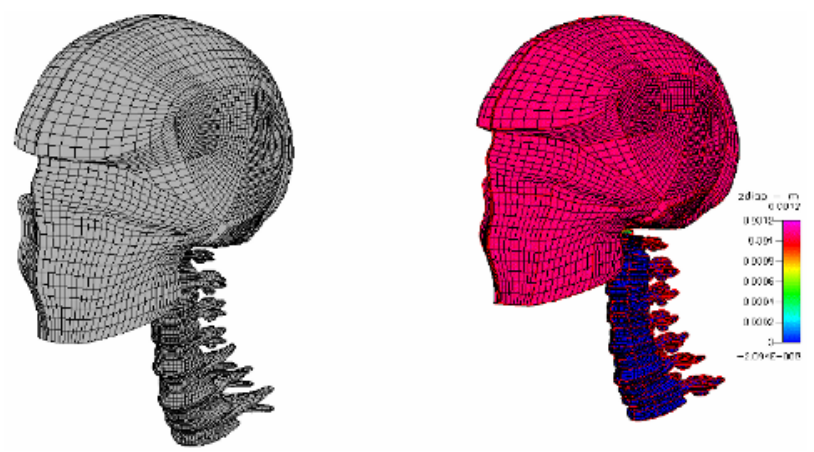

Fig. 8. The head-spine FE model and explicit solution for the head-spine model at $t=0.12 \mathrm{~ms}$

Table 1. CPU time and Memory requirements of explicit FE solvers for one time step

\begin{tabular}{|l|l|l|}
\hline Model Size (no. of dofs) & CPU (sec) & Memory (MB) \\
\hline 155,394 & 16.1 & 150 \\
\hline
\end{tabular}

\subsection{D Head-Spine Model under Blunt Impact}

To test the computational method a $2 \mathrm{D}$ multi-body test problem comprised of a helmet-head-cervical spine under rear impact has been setup. A rigid projectile is impacting the outer surface of the helmet with initial velocity of $30 \mathrm{~m} / \mathrm{s}$. The helmet, protection pad, head and C0-T1 spine are approximately modeled with 2D solid elements. The neck muscle and the ligament between vertebrae are modeled with the viscoelastic truss element. The bottom surface of T1 vertebrate is fixed. In Fig. 9, as the projectile is decelerated and rebounded during the impact on the helmet, both the 
helmet and the protection pad are deformed and rotated along the skull, and part of the absorbed energy by the helmet is transferred to the entire head in the form of rapid, typically angular acceleration of the head. Severe injuries of the skull, brain and cervical spine can be identified under this impact based on certain head injury criteria (HIC) such as the critical strain/strain rate at the tissue level.
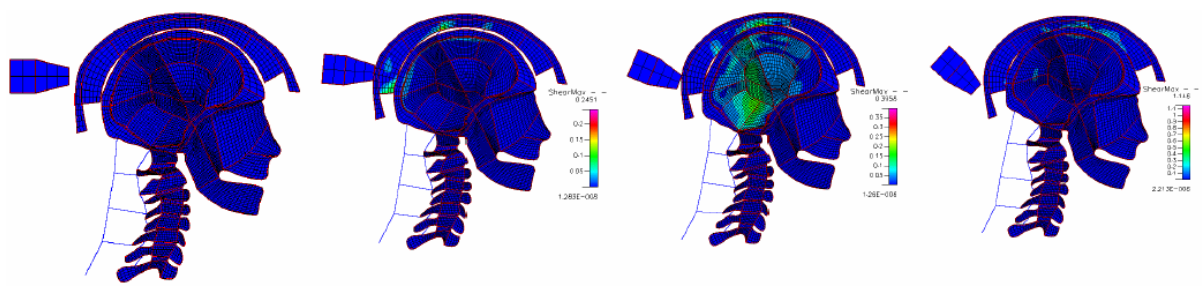

Fig. 9. Solid-Truss multi-body system under impact at time 1) $0 \mathrm{~ms}$, 2) $0.5 \mathrm{~ms}, 3) 1.2 \mathrm{~ms}$, 4) $1.8 \mathrm{~ms}$

It is noted that since we model the cerebrospinal fluid between the skull and the brain as the solid elements, the motion of the brain may be more constrained than in the real situation. We will report in the near future how the brain deforms surrounded by CSF modeled with the fluid elements.

\section{Discussions}

We demonstrated a simulation pipeline for modeling trauma to head and spine. We plan to develop robust, generic advanced modeling tools and experimental procedures for modeling trauma under ballistic and explosion blast injury. We would like to develop streamlined procedure to generate good quality FEM (volumetric) meshes for human organs from medical images. Databases are needed with examples of parametric head/neck geometries, and tissue specific material properties. The overall goal is to develop of tightly coupled FEM-CFD software tool for modeling a wide range of trauma cases.

\section{Acknowledgements}

We would like to thank Autodesk for generous donation of Maya Software, and Dr. Udupa from University of Pennsylvania for letting us use 3DVIEWNIX to model head anatomy.

\section{References}

1. Imielinska, C., Molholt, P., "Incorporating 3D Virtual Anatomy into the Medical Curriculum", $C A C M$, pp.49-54,Feb. 2005.

2. Imielinska C., Udupa, J.K., Metaxas, D., Jin, Y., Angelini, E., Chen, T., Zhuge, Y.,"Hybrid Segmentation Methods", book chapter in "Insight into Images: Principles and Practice for Segmentation, Registration, and Image Analysis", edited by T. Yoo, A.K. Peters, March 2004. 
3. Bathe, K.-J., "Finite Element Procedures", Prentice Hall, New Jersey, 1996.G.J.

4. Grevera, G.J., Udupa, J.K., Odhner, D., "An Order of Magnitude Faster Isosurface Rendering in Software on a PC than Using Dedicated, General Purpose Rendering Hardware", IEEE Trans. on Visualization and Computer Graphics, 6 (4), pp. 335-345, 2000.

5. Horgan, T.J., Gilchrist, M.D., The creation of three-dimensional finite element models for simulating head impact biomechanics. International Journal of Crashworthiness, 8 (4), pp. 353-366 (2003). 